\title{
Logarithmetics of Finite Quasigroups (I)
}

\author{
By Helen Popova
}

(Received 25th July, 1951.

Read 2nd November, 1951.)

\section{Introduction.}

The study of non-associative algebras led to the investigation of identities connecting powers of elements of such algebras. Thus Etherington ${ }^{1}$ (1941, 1949, 1951) introduced the concept of the logarithmetic of an algebra, defining it roughly as "the arithmetic of the indices of the general element".

Apart from a trivial observation on groups in $\$ 2$, the only known result concerning logarithmetics of quasigroups seems to be the result due to Murdoch $^{2}$ (1939, Corollary to Theorem 10). In Etherington's terminology this result is expressed by saying that an abelian quasigroup is palintropic, which means that multiplication is commutative in its logarithmetic $\left(x^{r s}=x^{s r}\right)$.

We introduce a new term quasi-integer; otherwise we follow Etherington in the definitions of $\S 2$.

I am grateful to Dr. I. M. H. Etherington for advice and criticisms.

\section{Definitions.}

A groupoid is a set closed with respect to a binary operation. A multiplicative groupoid with or without other operations such as + may be called an algebra. A (multiplicative) quasigroup ${ }^{3}$ means a multiplicative groupoid within which the equations $a x=b, y a=b$ determine $x$ and $y$ uniquely, whenever $a$ and $b$ are given; it is abelian (Murdoch, 1939) or entropic (Etherington, 1949) if identically $a b . c d=a c . b d$.

1 I. M. H. Etherington, "Some non-associative algebras in which the multiplication of indices is commutative", Journal London Math. Soc., 16 (1941), 48-55; "Nonassociative arithmetics", Proc. Roy. Soc. Edinburgh (A), 62 (1949), 442-453; "Noncommutative train algebras of rank 2 and 3 ", Proc. London Math. Soc. (2), 52 (1951), 241-252.

${ }^{2}$ D. C. Murdoch, "Quasigroups which satisfy certain generalised associative laws" American J. of Math., 61 (1939), 509-522.

3 B. A. Hausmann and O. Ore, "Theory of quasigroups", American .J. of Math., 59 (1937), 983-1004. 
A power $x^{r}$ of an element $x$ of an algebra $A$ is a continued product in which all factors are equal to $x$. The symbol $r$ used to denote the power is the index of the power. The product of two powers $x^{r}, x^{s}$ is denoted by $x^{r+s}$; a power of a power is indicated as a product in the index : $\left(x^{r}\right)^{s}=x^{r s}$; an iterated power is indicated by a power in the index: $\left(x^{r}\right)^{r}=x^{2}$, $\left(\left(x^{r}\right)^{r}\right)^{r}=x^{r^{s}}$, etc. For example

$$
x^{2.2+1}=\left(x^{2}\right)^{2} x ; \quad x^{(1+2.2) 2}=\left(x \cdot\left(x^{2}\right)^{2}\right)\left(x \cdot\left(x^{2}\right)^{2}\right) .
$$

The degree of a power of $x$ is the number of its factors $x$. Powers in which factors are absorbed one at a time on the right are called principal. The principal power of degree $\delta$ will be denoted $x^{\delta}$. All other powers can be expressed in terms of principal powers by suitably partitioning the index and using brackets when necessary. Thus $x^{4}=x^{(2+1)+1}$ is distinguished from $x^{1+3}=x^{1+(2+1)}$ and from $x^{1+(1+2)}$ and $x^{(1+2)+1}$.

A quasi-integer of an algebra $A$ will be defined as the class of indices $r, s, \ldots$ such that $x^{r}=x^{s}=\ldots$ for all $x$ of $A$.

It is easily seen that the quasi-integers can be added and multiplied like indices without inconsistency, and like indices they obey the rules ${ }^{1}$ :

$$
(r s) t=r(s t), \quad r(s+t)=r s+r t
$$

but in general

$$
r+(s+t) \neq(r+s)+t, \quad r+s \neq s+r, \quad r s \neq s r, \quad(s+t) r \neq s r+t r .
$$

The algebra consisting of all quasi-integers of $A$ together with operations $(+),($.$) is defined to be the logarithmetic of A$ and denoted by $L_{\mathbf{A}}$. Thus for example the logarithmetic of a commutative or associative algebra is commutative or associative with respect to addition; in particular the logarithmetic of a group with finite period $p$ is isomorphic with the ring of integers modulo $p$.

The set of all quasi-integers of $A$ together with the operation of addition or multiplication only will be denoted by $L_{\Lambda}(+), L_{A}($.) respectively.

Every subset of a finite quasigroup $Q$ which is closed with respect to multiplication satisfies the quotient axiom and is therefore a subquasigroup. In particular all powers of an element $a$ of $Q$ form a quasigroup $Q_{a}$. We

I. M. H. Etherington, " On non-ageociative combinations ", Proc. Roy. Soc. Edinburgh. 59

(1039), 153-162. 
shall' say that $Q_{a}$ is generated by $a$; its logarithmetic will be called the logarithmetic of $a$ and denoted by $L_{a}$.

\section{Quasi-integers of a finite algebra.}

A quasi-integer of an algebra $A$ consisting of a finite number of elements can be represented by the vector

$$
r=\left[\begin{array}{c}
a_{1}{ }^{r} \\
\vdots \\
a_{n}{ }^{r}
\end{array}\right],
$$

which sometimes will be written as:

$$
r=\left\{a_{p}^{r}\right\}_{p=1, \ldots, n} \text { or } r=\left\{a_{1}^{r}, \ldots, a_{n}^{r}\right\}
$$

where $a_{1}, \ldots, a_{n}$ are all elements (or, if preferred, all non-idempotent elements) of $A$. Two indices $r, s$ are equal in $L_{A}$ (i.e. belong to the same quasi-integer) if and only if $a_{i}^{r}=a_{i}{ }^{8}$ for $i=1,2, \ldots, n$, that is if and only if they are represented by the same vectors. If corresponding elements of two vectors $r=\left\{a_{p}^{r}\right\}$ and $s=\left\{a_{p}^{s}\right\} \quad(p=1,2, \ldots, n)$ are multiplied, we obtain $\left\{a_{p}^{r+s}\right\}$ which is the vector denoting $r+s$. The $s$-th powers of the elements of $r=\left\{a_{p}{ }^{r}\right\}_{p=1, \ldots, n}$ form the vector $\left\{a_{p}{ }^{r s}\right\}_{p=1, \ldots, n}$ which is $r s$. Consequently, if quasi-integers $r, s$ are given as $r=\left\{\lambda_{p}\right\}, s=\left\{\mu_{p}\right\}$ where $p=1,2, \ldots, n$, then

$$
r+s=\left\{\lambda_{p} \mu_{p}\right\}, \quad r s=\left\{\lambda_{p}{ }^{s}\right\}, \quad s r=\left\{\mu_{p}^{r}\right\} \quad(p=1,2, \ldots, n) .
$$

Multiplication in $L_{A}$ has an obvious matrix representation. If in the $k$-th row of the vector $r$ stands the element $a_{i}$ of $A$, then the element in the $k$-th row of the vector $r s$ is $a_{i}{ }^{s}$ which we find in the $i$-th row of the vector $s$. If we denote $a_{i}$ by a row vector with 1 in the $i$-th column and other elements zero:

$$
a_{i}=(0 \ldots 010 \ldots 0)
$$

and write vectors $r, s$ as matrices formed by substituting the vectors (2) in the expressions (1) of $r, s$, then $r s$ is the matrix product.

Example 1. Investigating the logarithmetic of the quasigroup $Q$ consisting of elements $1,2,3,4$, given by the multiplication table

\begin{tabular}{l|llll}
\multicolumn{1}{c}{1} & \multicolumn{1}{c}{1} & 2 & 3 & 4 \\
\cline { 2 - 6 } 1 & 3 & 1 & 4 & 2 \\
2 & 4 & 2 & 1 & 3 \\
3 & 1 & 3 & 2 & 4 \\
4 & 2 & 4 & 3 & 1
\end{tabular}


we observe that any quasi-integer of $L_{0}$ can, since 2 is idempotent, be completely determined by the set of elements $\left(1^{r}, 3^{r}, 4^{r}\right)=(m, n, s)$, where $m, n, s$ can take any values amongst $1,2,3,4$. Thus :

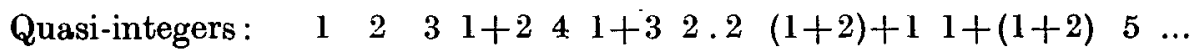

Elements of $Q$ :

\begin{tabular}{|c|c|c|c|c|c|c|c|c|}
\hline 1 & 3 & 1 & 4 & 3 & 3 & 2 & 2 & 2 \\
\hline 3 & 2 & 1 & 3 & 4 & 1 & 2 & 2 & 2 \\
\hline 4 & 1 & 2 & 2 & 3 & 4 & 3 & 3 & 4 \\
\hline
\end{tabular}

and we may denote quasi-integers of $L_{Q}$ by vectors such as

$$
\begin{aligned}
& 1=\left[\begin{array}{l}
1 \\
3 \\
4
\end{array}\right]=(1+3)+3 ; \quad(1+2)=\left[\begin{array}{l}
4 \\
3 \\
2
\end{array}\right] ; 2.2=\left[\begin{array}{l}
2 \\
2 \\
3
\end{array}\right] ; \quad 2=\left[\begin{array}{l}
3 \\
2 \\
1
\end{array}\right] ; \\
& 1+(1+2)=\left[\begin{array}{l}
2 \\
2 \\
4
\end{array}\right] ; \ldots .
\end{aligned}
$$

It may be verified that the 64 such vectors all occur in $L_{Q}$.

Example 2. Suppose that $r=\{3,2,1,4\} s=\{3,2,4,3\}$. (This could refer to the logarithmetic of Ex. 1, with $r=1+3, s=4$, since the element 2 is idempotent.) Then we have $1^{s}=3,2^{s}=2,3^{s}=4,4^{s}=3$, giving

$$
r s=\left\{3^{8} ; 2^{s}, 1^{s}, 4^{s}\right\}=\{4,2,3,3\} .
$$

As in the previous section, denoting the elements $1,2,3,4$ of $Q$ by row vectors $(1 \ldots),(.1 \ldots),(\ldots 1),.(\ldots 1)$ respectively, we can write the column vectors $r, s$ as matrices. In this notation

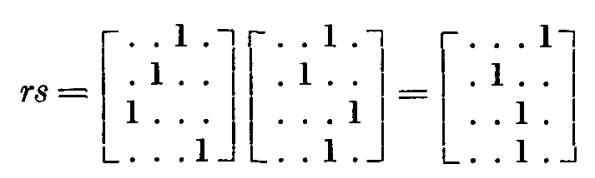

4. Properties of $L_{Q}(+)$.

Let $L_{i}(i=1,2, \ldots)$ be any finite or infinite set of algebras, distinct or identical, with operations $(+),($.$) uniquely defined by$

$$
q_{i}+p_{i}=r_{i}, \quad q_{i} p_{i}=t_{i}, \quad q_{i}, p_{i}, r_{i}, t_{i} \varepsilon L_{i} \quad(i=1,2, \ldots)
$$

and consider the set $L^{\times}$of all symbols

$$
\left\{q_{1}, q_{2}, \ldots\right\}, \quad q_{i} \in L_{i} \quad(i=1,2, . .)
$$


with operations $(+),($.$) defined as$

$$
\left\{q_{1}, \ldots\right\}+\left\{p_{1}, \ldots\right\}=\left\{r_{1}, \ldots\right\}, \quad\left\{q_{1}, \ldots\right\}\left\{p_{1}, \ldots\right\}=\left\{t_{1}, \ldots\right\} ;
$$

then $L^{\times}$is called the direct union ${ }^{1}$ of $L_{1}, L_{2}, \ldots$

LEMMA. The direct union $L^{\times}$of the logarithmetics $L_{i}$ of all the elements $1,2, \ldots, n$ of a finite quasigroup is a finite quasigroup with respect to addition.

For the elements of $L^{\times}$are vectors such as

$$
r=\left\{p^{r_{r}}\right\}, \quad s=\left\{p^{s}\right\} \quad(p=1,2, \ldots, n) .
$$

Obviously $r+s=\left\{p^{r_{p}+s_{p}}\right\}$ belongs to $L^{\times}$, and it remains to prove that the equations $r+x=s, y+r=s$ always have unique solutions $x, y$ in $L^{\times}$.

Now $r+x=s$ is equivalent to the set of $n$ equations

$$
p^{r_{p}} x_{p}=p^{s_{r}} .
$$

Since all powers of $p$ form a quasigroup, each of these equations has a unique solution of the form $x_{p}=p^{x}$. Thus $r+x=s$ has the unique solution $x=\left\{p^{x_{p}}\right\}$, which is in $L^{\times}$. Similarly for $y+r=s$.

TheOREM 1. The logarithmetic of a finite quasigroup is a quasigroup with respect to addition.

For the vectors of $L_{Q}$, say $r=\left\{p^{r}\right\}, s=\left\{p^{s}\right\}, p=1, \ldots, n$, may also be regarded as vectors of $L^{\times}$. Thus the logarithmetic of $Q$ is a subset of a finite additive quasigroup $L^{\times}$, closed with respect to addition, and therefore is a quasigroup with respect to addition.

Example 3. The quasigroup $Q$ of order four

\begin{tabular}{l|llll}
\multicolumn{1}{c}{} & \multicolumn{1}{l}{1} & 2 & 3 & 4 \\
\cline { 3 - 5 } 1 & 2 & 4 & 3 & 1 \\
2 & 3 & 1 & 2 & 4 \\
3 & 1 & 3 & 4 & 2 \\
4 & 4 & 2 & 1 & 3
\end{tabular}

has logarithmetic consisting of only four quasi-integers

$$
1=\left[\begin{array}{l}
1 \\
2 \\
3 \\
4
\end{array}\right], \quad 2=\left[\begin{array}{l}
2 \\
1 \\
4 \\
3
\end{array}\right], \quad 3=\left[\begin{array}{l}
3 \\
4 \\
1 \\
2
\end{array}\right], \quad 1+2=\left[\begin{array}{l}
4 \\
3 \\
2 \\
1
\end{array}\right] \text {. }
$$

In this case $L_{Q}(+)$ is isomorphic with $Q$.

1 G. Birkhoff, "On the structure of abstract algebras", Proc. Oambridge Phil. Soc., 31 (1935), 433-454. 


\section{5. $L_{Q}$ as a subdirect union.}

Let $L^{\times}$be a direct union of an arbitrary set of additive quasigroups $L_{i}$, the elements of $L^{\times}$being denoted by

$$
l=\left\{\alpha_{1}, \alpha_{2}, \ldots, \alpha_{n}\right\}, \quad \alpha_{i} \varepsilon L_{i}
$$

If $L$ is a subquasigroup of $L^{\times}$, and

$$
q=\left\{q_{1}, q_{2}, \ldots, q_{n}\right\} \in L,
$$

the correspondence $q \rightarrow q_{i}$ defines a homomorphism of $L$ into $L_{i}$ and therefore on to a subquasigroup $L_{i}^{\prime}$ of $L_{i}$. If for every $i L_{i}^{\prime}=L_{i}, L$ is a subdirect union of the quasigroups $L_{i}$.

Let $Q$ be a quasigroup $(1,2, \ldots, n)$. We denote by $L_{i}$ the logarithmetic of the element $i$ of $Q(i=1,2, \ldots, n)$.

Let $i^{x}$ take $n_{i}$ distinct values $\beta_{i 1}, \beta_{i 2}, \ldots, \beta_{i n_{i}}$ when $x$ varies $(i=1,2, \ldots, n)$. The direct union

$$
L^{\times}=L_{1}+L_{2}+\ldots+L_{n}
$$

consists of all $n_{1} n_{2} \ldots n_{n}$ possible vectors

$$
\left\{a_{1}, a_{2}, \ldots, a_{n}\right\} \text { where } a_{i} \varepsilon L_{i} \text {. }
$$

The logarithmetic of $Q$ does not necessarily contain all those vectors. However (Theorem 1), it forms a quasigroup with respect to addition, which is a subquasigroup of $L^{\times}$.

All the vectors representing the quasi-integers of $L_{Q}$ may be written in a matrix

$$
L=\left[\begin{array}{ccc}
\alpha_{11} & \ldots & \alpha_{1 N} \\
\ldots & \ldots & \ldots \\
\alpha_{n 1} & \ldots & \alpha_{n N}
\end{array}\right]
$$

where $n$ is the order of $Q, N$ that of $L_{Q}$, and $\alpha_{i j} \varepsilon L_{i}$.

From the fact that $L_{Q}$ is the set of all distinct values of $\{1, \ldots, n\}^{x}$ when $x$ is varied, it follows that in the $i$-th row of the matrix $L$ there appear necessarily all distinct elements of $L_{i}$. Therefore, if we collect the quasiintegers with $\beta_{i 1}, \beta_{i 2}, \ldots, \beta_{i n_{i}}$ in the $i$-th row into classes $A_{i 1}, \ldots, A_{i n}$ respectively, the homomorphisms $q \rightarrow q_{i}$ above are

$$
A_{i 1} \rightarrow \beta_{i 1}, A_{i 2} \rightarrow \beta_{i 2}, \ldots, A_{i n_{i}} \rightarrow \beta_{i n_{i}} \quad(i=1,2, \ldots, n) .
$$

Each of them defines the homomorphism of $L_{Q}$ on to $L_{i}$

$$
L_{Q} \rightarrow L_{i} \quad(i=1,2, \ldots, n)
$$


and we have proved:

ThEOREM 2. The logarithmetic of a quasigroup is a subdirect union of the logarithmetics of its elements.

By the order $n_{i}$ of the element $i$ of a quasigroup $Q$ we understand the order of the quasigroup generated by it.

Corollary 1. The order of $L_{a}$ cannot exceed the product of the orders of all elements of $Q$ :

$$
N \leqslant n_{1} n_{2} \ldots n_{n} \text {. }
$$

For $n_{1} n_{2} \ldots n_{n}$ is the order of the direct union.

Corolcary 2. If $L_{Q}$ has order $N=n_{1} n_{2} \ldots n_{n}$, then it is the direct union of the logarithmetics of all elements of $Q$.

(Compare Example 1.)

Example 4. The logarithmetic of the quasigroup.

\begin{tabular}{l|llll}
\multicolumn{1}{c}{} & \multicolumn{1}{l}{1} & 2 & 3 & 4 \\
\cline { 2 - 5 } 1 & 2 & 3 & 4 & 1 \\
2 & 4 & 1 & 2 & 3 \\
3 & 3 & 2 & 1 & 4 \\
4 & 1 & 4 & 3 & 2
\end{tabular}

consists of 16 quasi-integers which are the columns of the matrix

$$
L=\left[\begin{array}{llllllllllllllll}
1 & 1 & 1 & 1 & 2 & 2 & 2 & 2 & 3 & 3 & 3 & 3 & 4 & 4 & 4 & 4 \\
2 & 2 & 2 & 2 & 1 & 1 & 1 & 1 & 4 & 4 & 4 & 4 & 3 & 3 & 3 & 3 \\
1 & 2 & 3 & 4 & 1 & 2 & 3 & 4 & 1 & 2 & 3 & 4 & 1 & 2 & 3 & 4 \\
2 & 1 & 4 & 3 & 2 & 1 & 4 & 3 & 2 & 1 & 4 & 3 & 2 & 1 & 4 & 3
\end{array}\right]
$$

The logarithmetics of the elements $1,2,3,4$ are

$$
L_{1}=(1,2,3,4), \quad L_{2}=(1,2,3,4), \quad L_{3}=(1,2,3,4), \quad L_{4}=(1,2,3,4) .
$$

So the direct union consists of 256 vectors. The logarithmetic, however, has order 16, and the homomorphisms $q \rightarrow q_{l}$ are :

(1) $q \rightarrow q_{1}$ :

$$
\left[\begin{array}{llll}
1 & 1 & 1 & 1 \\
2 & 2 & 2 & 2 \\
1 & 2 & 3 & 4 \\
2 & 1 & 4 & 3
\end{array}\right] \rightarrow 1,\left[\begin{array}{llll}
2 & 2 & 2 & 2 \\
1 & 1 & 1 & 1 \\
1 & 2 & 3 & 4 \\
2 & 1 & 4 & 3
\end{array}\right] \rightarrow 2,\left[\begin{array}{llll}
3 & 3 & 3 & 3 \\
4 & 4 & 4 & 4 \\
1 & 2 & 3 & 4 \\
2 & 1 & 4 & 3
\end{array}\right] \rightarrow 3,\left[\begin{array}{llll}
4 & 4 & 4 & 4 \\
3 & 3 & 3 & 3 \\
1 & 2 & 3 & 4 \\
2 & 1 & 4 & 3
\end{array}\right] \rightarrow 4
$$


which implies $L_{Q} \rightarrow L_{1}$. Similarly

(2) $q \rightarrow q_{2}$ :

$$
\left[\begin{array}{llll}
2 & 2 & 2 & 2 \\
1 & 1 & 1 & 1 \\
1 & 2 & 3 & 4 \\
2 & 1 & 4 & 3
\end{array}\right] \rightarrow 1,\left[\begin{array}{llll}
1 & 1 & 1 & 1 \\
2 & 2 & 2 & 2 \\
1 & 2 & 3 & 4 \\
2 & 1 & 4 & 3
\end{array}\right] \rightarrow 2,\left[\begin{array}{llll}
4 & 4 & 4 & 4 \\
3 & 3 & 3 & 3 \\
1 & 2 & 3 & 4 \\
2 & 1 & 4 & 3
\end{array}\right] \rightarrow 3,\left[\begin{array}{llll}
3 & 3 & 3 & 3 \\
4 & 4 & 4 & 4 \\
1 & 2 & 3 & 4 \\
2 & 1 & 4 & 3
\end{array}\right] \rightarrow 4
$$

(3) $q \rightarrow q_{3}$ :

$$
\left[\begin{array}{llll}
1 & 2 & 3 & 4 \\
2 & 1 & 4 & 3 \\
1 & 1 & 1 & 1 \\
2 & 2 & 2 & 2
\end{array}\right] \rightarrow 1,\left[\begin{array}{llll}
1 & 2 & 3 & 4 \\
2 & 1 & 4 & 3 \\
2 & 2 & 2 & 2 \\
1 & 1 & 1 & 1
\end{array}\right] \rightarrow 2,\left[\begin{array}{llll}
1 & 2 & 3 & 4 \\
2 & 1 & 4 & 3 \\
3 & 3 & 3 & 3 \\
4 & 4 & 4 & 4
\end{array}\right] \rightarrow 3,\left[\begin{array}{llll}
1 & 2 & 3 & 4 \\
2 & 1 & 4 & 3 \\
4 & 4 & 4 & 4 \\
3 & 3 & 3 & 3
\end{array}\right] \rightarrow 4
$$

(4) $q \rightarrow q_{4}$ :

$$
\left[\begin{array}{llll}
1 & 2 & 3 & 4 \\
2 & 1 & 4 & 3 \\
2 & 2 & 2 & 2 \\
1 & 1 & 1 & 1
\end{array}\right] \rightarrow 1,\left[\begin{array}{llll}
1 & 2 & 3 & 4 \\
2 & 1 & 4 & 3 \\
1 & 1 & 1 & 1 \\
2 & 2 & 2 & 2
\end{array}\right] \rightarrow 2, \quad\left[\begin{array}{llll}
1 & 2 & 3 & 4 \\
2 & 1 & 4 & 3 \\
4 & 4 & 4 & 4 \\
3 & 3 & 3 & 3
\end{array}\right] \rightarrow 3,\left[\begin{array}{llll}
1 & 2 & 3 & 4 \\
2 & 1 & 4 & 3 \\
3 & 3 & 3 & 3 \\
4 & 4 & 4 & 4
\end{array}\right] \rightarrow 4,
$$

which shows that the homomorphisms $q \rightarrow q_{2}, q \rightarrow q_{3}, q \rightarrow q_{4}$ imply the homomorphisms

$$
L_{Q} \rightarrow L_{2}, \quad L_{Q} \rightarrow L_{3}, \quad L_{Q} \rightarrow L_{4}
$$

respectively. So that, for every $i, L_{i}^{\prime}=L_{i}$, and $L_{Q}$ is a subdirect union of $L_{1}, L_{2}, L_{3}$ and $L_{4}$.

Department of Mathematics, UNIVERSITY OF ABERDEEN. 